Philosophia

Scientie

\title{
Philosophia Scientiæ
}

Travaux d'histoire et de philosophie des sciences

23-2 | 2019

Expérimentation dans les sciences de la nature Expérimentation dans les sciences humaines et sociales

\section{Introduction. Philosopher sur l'expérimentation scientifique : bilan et perspectives}

Introduction. Philosophizing about scientific experimentation: a summary report and future prospects

Catherine Allamel-Raffin, Stéphanie Dupouy et Jean-Luc Gangloff

\section{(2) OpenEdition \\ 1 Journals}

\section{Édition électronique}

URL : http://journals.openedition.org/philosophiascientiae/1899

DOI : 10.4000/philosophiascientiae.1899

ISSN : $1775-4283$

Éditeur

Éditions Kimé

Édition imprimée

Date de publication : 24 mai 2019

Pagination : $5-18$

ISBN : 978-2-84174-933-1

ISSN : 1281-2463

Référence électronique

Catherine Allamel-Raffin, Stéphanie Dupouy et Jean-Luc Gangloff, «Introduction. Philosopher sur l'expérimentation scientifique : bilan et perspectives », Philosophia Scientiæ [En ligne], 23-2 | 2019, mis en ligne le 01 janvier 2021, consulté le 30 mars 2021. URL : http://journals.openedition.org/ philosophiascientiae/1899; DOI : https://doi.org/10.4000/philosophiascientiae.1899 


\section{Introduction. Philosopher sur l'expérimentation scientifique : bilan et perspectives}

Stéphanie Dupouy

Université de Strasbourg, Archives Henri-Poincaré Philosophie et Recherches sur les Sciences et les Technologies (AHP-PReST, UMR 7117) (France)

Jean-Luc Gangloff

Université de Strasbourg, Archives Henri-Poincaré Philosophie et Recherches sur les Sciences et les Technologies (AHP-PReST, UMR 7117) (France)

Le projet à l'origine de ce dossier thématique est celui d'une étude comparative de l'expérimentation telle qu'elle apparaît dans les sciences de la nature et dans les sciences humaines et sociales. Il illustre et prolonge les réflexions d'un séminaire de recherche sur le même sujet, organisé par Catherine AllamelRaffin, qui s'est tenu pendant deux ans (2017-2018) à l'université de Strasbourg grâce à un financement de la Misha (Maison Interuniversitaire des Sciences de l'Homme - Alsace). Les pages qui suivent présentent quelques-uns des constats et des propositions qui ont constitué le point de départ de ce travail collectif. 


\section{Bref historique de la recherche sur l'expérimentation au sein de la philosophie générale des sciences}

Parallèlement à celle des historiens et des sociologues, la contribution des philosophes à la réflexion sur l'expérimentation scientifique a été importante tout au long du $\mathrm{xx}^{\mathrm{e}}$ siècle. Durant la première moitié de celui-ci, l'expérimentation (ou plutôt le couple observation/expérimentation, les deux concepts étant alors englobés sous un concept plus général d'épreuve ou de confirmation empirique) s'est vue conférer une fonction épistémologique centrale, celle de fondement de la connaissance scientifique, lui permettant de se démarquer de la métaphysique ou des pseudosciences. La place prépondérante accordée à l'expérimentation ainsi qu'à l'observation par les positivistes logiques et par Popper, dans les processus de vérification ou de corroboration-réfutation des hypothèses, l'a toutefois été dans le cadre restrictif d'une méthodologie déterminée, à savoir l'analyse logique du langage scientifique [Carnap 1934], [Popper 1934], [Nagel 1961]. Une telle démarche, féconde à son échelle, pouvait inciter à se pencher sur des questions telles que celles de la nature ou du statut des énoncés protocolaires par exemple. Elle négligeait en revanche de mettre en œuvre une réflexion approfondie sur les pratiques expérimentales effectives - et ce de manière légitime en vertu de l'agenda philosophique qui la sous-tendait et ne l'orientait pas dans cette direction. La philosophie des sciences de la fin $\mathrm{du} \mathrm{XX}^{\mathrm{e}}$ siècle, quant à elle, a peu à peu restitué son épaisseur et sa complexité au moment expérimental, jusqu'alors réduit à un simple point de contact, instantané et passif, avec la nature. Elle va s'intéresser aux savoir-faire, aux ajustements progressifs, et aux décisions que l'expérimentation met en ouvre, ainsi qu'à l'environnement matériel et social qui la rendent possible.

Comme on le sait, cette place de l'expérimentation en tant qu'objet d'étude au sein de la philosophie des sciences a été reconsidérée en deux étapes au cours $\mathrm{du} \mathrm{Xx}^{\mathrm{e}}$ siècle. La première étape a revêtu la forme de ce que l'on a appelé le «tournant historique », initié notamment par l'ouvrage de Thomas S. Kuhn [Kuhn 1962] qui recommandait l'abandon de la méthodologie normative au profit de l'étude des communautés de scientifiques et des paradigmes au sein desquels ceux-ci développent leurs recherches. Dans les années 1980, le « tournant pratique » [Soler, Zwart et al. 2014], dans le cadre duquel Ian Hacking a joué un rôle moteur, a permis de préciser, au travers de nombreuses études de cas, en quel sens on peut considérer que « l'expérimentation a sa vie propre» [Hacking 1983, 150]. Les travaux entrepris alors ont eu des visées multiples. L'une d'entre elles a consisté à réévaluer la subordination de l'expérience à la théorie généralement admise par les philosophes des sciences (aussi bien dans la tradition positiviste que dans l'héritage de Kuhn qui insistait sur la charge théorique des observations), avec pour finalité ultime de rendre compte de la 
diversité des modes d'interaction entre théorie et expérience et de la variété des styles de raisonnement scientifique :

En aucune manière je n'essaie de valoriser l'expérience au détriment de la théorie. Je cherche avant tout à faire voir la richesse, la complexité et la variété de la vie scientifique. La théorie n'est pas une, mais multiple, et l'expérimentation n'est pas une, mais multiple. La philosophie des sciences a été appauvrie non seulement par son obsession de la théorie, mais aussi par l'idée orgueilleuse et vaine qu'il n'y aurait, si ce n'est un seul, au plus deux types de théories, par exemple les vraies théories [real theories] et les principes de liaison [bridge principles]. Le mouvement de libération de l'expérimentation a eu non seulement pour but de rendre compréhensible l'activité expérimentale, mais aussi d'améliorer les conditions de réception des théories - tout en rendant la distinction théorie/expérience, si ce n'est obsolète, certainement moins univoque. [Hacking 2005, 68-69]

La lignée de philosophes des sciences s'inscrivant dans cette perspective (parfois réunis sous l'étiquette de " nouvel expérimentalisme») a effectivement tenté de saisir la complexité des relations entre théories et expérience et a mis l'accent sur la diversité des fonctions de l'expérimentation tout en répertoriant les ressources mobilisées dans le cadre de cette dernière [Galison 1987, 1997], [Franklin 1986, 1990, 2016], [Hon 1989, 2003], [Radder 2003], [Galavotti 2004]. Quelles sont les contraintes théoriques et expérimentales à l'œuvre dans le travail des chercheurs au sein du laboratoire? Quel statut épistémologique convient-il de conférer à l'instrumentation [Baird 2004], [Harré 2003] ? Quels sont plus généralement les constituants de l'investigation empirique dans les sciences [Franklin 1986, 1990, 2016], [Hacking 1992], [Hon 1989, 2003] ? Parallèlement à ces interrogations nouvelles traitées par les philosophes des sciences, la question de l'expérimentation a été abordée par des auteurs appartenant au courant des " études sociales sur la science» (ESS) [Collins 1985], [Gooding, Pinch et al. 1989], [Latour \& Woolgar 1979], [Pickering 1984, 1992, 1995]. Les conclusions des études de cas réalisées par ces auteurs ont visé à récuser le principe selon lequel une preuve empirique en faveur d'une hypothèse peut constituer un argument décisif dans le cadre d'une entreprise de confirmation ou de réfutation de cette dernière. La perspective plus large dans laquelle s'inscrivent les travaux des ESS est celle d'une remise en question d'une conception atemporelle et désintéressée de la rationalité, au profit d'une vision de la recherche scientifique comme essentiellement déterminée par le contexte historique et social. Des lignes de fracture sont de ce fait apparues entre nouveaux expérimentalistes et auteurs des ESS, et le débat s'est déplacé d'une réflexion sur la place de l'expérimentation dans le cadre des processus d'investigation scientifique, à l'affirmation ou au rejet d'un relativisme épistémique plus ou moins assumé, celui-ci prenant pour argument le caractère 
jugé non déterminant de certaines expérimentations effectives, observées par les sociologues, dans la justification des résultats.

Ces dissensions n'ont pas cessé à ce jour. Or, on peut remarquer que, sauf exception, les termes du débat ont toujours été posés au sein d'un champ d'investigation empirique limité aux sciences de la nature [Pickering 1984], [Collins 2001], [Allamel-Raffin \& Gangloff 2015], [Soler, Zwart et al. 2014]. La nature même de la controverse justifiait en effet ici de partir des sciences expérimentales (la physique au premier chef) dans lesquelles l'idéal de falsifiabilité empirique, pour la conception positiviste classique, paraissait le plus assuré. De ce fait, sans constituer délibérément un point aveugle, l'expérimentation dans les sciences humaines et sociales est donc très largement restée hors champ de ces controverses entre philosophes et sociologues des sciences.

\section{L'épistémologie des sciences humaines et sociales et la question de l'expérimentation}

Bien qu'elle constitue aujourd'hui un domaine de recherche en pleine expansion, l'épistémologie générale des sciences humaines et sociales n'a pas non plus fait de l'expérimentation (ni même de l'observation) un point focal de ses analyses. Faut-il rappeler d'abord que bon nombre de disciplines des sciences humaines n'utilisent pas du tout l'expérimentation ou ne le font que très marginalement? L'épistémologie des sciences humaines a donc généralement privilégié d'autres objets et niveaux d'analyse : par exemple les débats concernant le mode d'intelligibilité propre à ces sciences - leur nature interprétative ou explicative; le problème de savoir si leur visée dernière consiste à connaître des généralités ou des singularités; les questions d'ontologie relatives au mode d'existence des entités mentales ou collectives; le bien-fondé du postulat de la rationalité comme réquisit explicatif du comportement humain; ou encore l'objectivité qu'il convient d'attribuer aux sciences humaines et sociales, plus susceptibles encore que les sciences de la nature de confondre l'empirique et le normatif, la science et la politique [Ogien 2001]. La question de l'expérimentation se trouve alors parfois posée dans ce champ sous d'autres rubriques, par exemple dans le cadre d'une réflexion sur la capacité des sciences humaines à tester leurs assertions [Popper 1965], ou sur les effets d'interaction entre observateurs et sujets observés - prophéties auto-réalisatrices, effets de boucle [Martin 1994]. De manière générale, le tournant pratique de la philosophie des sciences n'a encore rencontré qu'assez peu d'échos en épistémologie générale des sciences humaines et sociales ${ }^{1}$. En témoigne par exemple l'absence habituelle d'entrée « expérimentation »

1. Ian Hacking constitue là encore une exception [Hacking 1999]. 
dans les dictionnaires de philosophie des sciences sociales ou dans les manuels classiques d'épistémologie des sciences sociales ${ }^{2}$.

$\mathrm{Au}$ sein de telle ou telle discipline des sciences humaines, en revanche, la thématique qui fait l'objet de ce numéro a donné lieu à des réflexions approfondies. Les praticiens de certaines de ces disciplines ont en effet souvent, et parfois de longue date, produit des réflexions méthodologiques riches et variées sur la question de savoir si leur science était ou non susceptible d'utiliser la méthode expérimentale, sur les différences de cette méthode avec l'observation, s'il existait plusieurs formes, plus ou moins littérales, de méthodes expérimentales, ou si l'expérimentation posait des biais particuliers (ou nécessitait des précautions spécifiques) dans leur discipline par rapport aux sciences de la nature. Plus généralement, ils se sont parfois intéressés à la « bonne manière », pour leur discipline, de conduire une expérimentation ou aux ficelles du métier d'expérimentateur - ceci étant également vrai, mutatis mutandis, des pratiques d'observation. La psychologie, notamment, devenue (pour partie) expérimentale dès le milieu du XIX ${ }^{\mathrm{e}}$ siècle, a non seulement développé une longue tradition de réflexion sur l'expérimentation psychologique, mais présente l'originalité d'avoir fait de cette question un objet d'étude lui-même expérimental, par exemple dans les travaux précoces d'Alfred Binet sur la suggestibilité du sujet d'expérience [Binet 1900], ou dans les recherches systématiques entreprises, dans le sillage de la psychologie sociale des années 1970, par des psychologues expérimentalistes tels que Martin Orne ou Robert A. Rosenthal [Orne 1962], [Rosenthal, Jacobson et al. 1971] sur les biais de la situation expérimentale en psychologie. La sociologie, quant à elle, a d'emblée défini sa méthode d'enquête par comparaison avec la méthode expérimentale des sciences de la nature, en la caractérisant, chez Emile Durkheim notamment [Durkheim 1894], comme un substitut de méthode expérimentale [post hoc] et reposant sur l'utilisation de statistiques - avant de commencer à recourir aussi à l'expérimentation de terrain dans les années vingt et trente du $\mathrm{Xx}^{\mathrm{e}}$ siècle siècle [Mayo 1933].

2. Par exemple, pour les manuels : [Hollis 2002], [Berthelot 2001]. Dans ce dernier, le mot « expérimentation » apparaît seulement à trois reprises (dans les 594 pages de l'ouvrage), dans un article portant sur la science économique. Il est vrai que l'adjectif « expérimental», en revanche, est présent à cinq reprises, en particulier dans un chapitre qui contient une présentation par Berthelot lui-même de ce qu'il appelle « un modèle de scientificité commun fondé sur la raison expérimentale » [Berthelot 2001, 217]. Dans le volume Philosophy of Anthropology and Sociology [Turner \& Risjord 2007], paru dans la collection Handbook of the Philosophy of Science, le mot « experiment» n'apparaît qu'une fois sur l'ensemble des 884 pages du volume et figure dans un article consacré à la théorie de la mesure en psychologie. Enfin, dans le Dictionnaire des sciences humaines [Mesure \& Savidan 2006], sur l'ensemble de ses 1280 pages, on trouve cinq références seulement aux termes appartenant au champ lexical de l'expérimentation : de surcroît, celles-ci apparaissent le plus souvent dans des articles consacrés à des thèmes tels que « Biologie et société » (« expérimentation animale » et " expérimentation in vitro »). L'expérimentation, en tant qu'objet de réflexion intrinsèquement pertinent pour l'épistémologie des sciences humaines et sociales, ne donne pas lieu à un article qui lui serait exclusivement consacré. 
L'applicabilité du modèle expérimental à la sociologie a depuis suscité une riche réflexion épistémologique chez les sociologues [Passeron 1991]. Au milieu du $\mathrm{Xx}^{\mathrm{e}}$ siècle, la linguistique et l'économie sont venues grossir les rangs des sciences humaines pratiquant des expérimentations de laboratoire [Serra 2015] ; l'économie a elle aussi pratiqué l'expérimentation de terrain de manière massive dans les années 1960-1970 [Monnier 1992], [Gueron \& Rolston 2013], cette méthode revenant en force aujourd'hui à travers la promotion des ERC (Essais Randomisés Contrôlés) dans le domaine de l'économie du développement impulsée notamment par les économistes de J-PAL, le laboratoire d'Esther Duflo [Duflo 2009]. Plus récemment enfin, la philosophie morale elle-même a commencé à recourir à des formes diverses d'expérimentations (expériences de pensée ou expériences de laboratoire) [Knobe \& Nichols 2008], [Ogien 2011]. Il n'est pas jusqu'aux sciences historiques à n'intégrer aujourd'hui les pratiques expérimentales à leur arsenal méthodologique, qu'il s'agisse de la fabrication d'objets selon les savoir-faire anciens en histoire des techniques et archéologie [Monteix \& Poidevin 2019], ou des expériences de pensée pratiquées par l'histoire contrefactuelle [Bourgeois-Gironde 2012]. Si cette diffusion de la méthodologie expérimentale (sous des formes très variées) procède parfois d'un idéal naïf d'imitation des sciences de la nature, de la conviction noninterrogée que l'expérimentation constituerait nécessairement la voie royale de toute science, ou encore de stratégies plus obscures liées à la recherche de financements, elle a également favorisé l'analyse méthodologique et épistémologique fine des particularités de l'expérimentation dans ces disciplines elles-mêmes (ou chez les philosophes des sciences qui s'y intéressent ${ }^{3}$ ).

\section{Qu'appelle-t-on « expérimentation »?}

Une question liminaire s'est posée dans notre travail : à quelles conditions une pratique de recherche en sciences humaines et sociales peut-elle légitimement être qualifiée d'expérimentale? En fonction de ce que l'on acceptera ou non d'introduire dans ce champ, on sera amené à ajouter ou à refuser des caractéristiques dans la définition en intension. Classiquement, l'expérimentation suppose l'intervention délibérée dans le cours des phénomènes, et la manipulation contrôlée de ces phénomènes, notamment en vue de déterminer quels paramètres sont pertinents quant à la production d'un effet donné [Nadeau 1999, 235], [Soler 2009, 64], [Dupouy 2011, 213]. En ce sens, l'expérimentation diffère de l'observation par le fait que le chercheur opère une modification par rapport au déroulement ordinaire des événements. Par conséquent, et comme le remarquait déjà Claude Bernard [Bernard 1865], le critère pertinent qui permet de distinguer l'expérimentation de l'observation n'est pas constitué par le couple activité/passivité, mais par le fait que la première suppose la

3. Pour des exemples empruntés au développement des expérimentations aléatoires en économie, voir [Labrousse 2010], [Cartwright \& Hardy 2013]. 
création d'une situation artificielle, tandis que la seconde tend à laisser (dans la mesure du possible) toutes choses en l'état. En s'inspirant des analyses classiques de John Stuart Mill et d'Ernest Nagel, on pourrait ainsi énoncer trois conditions à satisfaire pour qu'une expérimentation puisse avoir lieu :

La première est que le chercheur puisse manipuler à volonté certaines variables considérées comme pertinentes pour produire les phénomènes qui l'intéressent; la seconde, que ces variables puissent être manipulées isolément les unes des autres; la troisième, que ces manipulations et leurs effets soient reproductibles. [Dupouy 2011, 216]

La distinction entre expérimentation et observation s'est également appuyée sur leur rôle dans le raisonnement causal : alors que l'observation permettrait de trouver les causes (en remontant des effets aux causes), l'expérimentation, par la méthode des différences [sublata causa, tollitur effectus], permettrait de prouver les causes (en allant des causes aux effets). Cette définition de l'expérimentation au sens strict peut être distinguée de ce que Claude Bernard appelle plus largement le « raisonnement expérimental » : il «n'est rien d'autre qu'un raisonnement à l'aide duquel nous soumettons méthodiquement nos idées à l'expérience des faits » [Bernard 1865, 103]. Le raisonnement expérimental est de ce fait absolument le même dans les sciences d'observation et les sciences expérimentales [Bernard 1865, 125].

Les sciences humaines et sociales se livrent-elles à des expérimentations au sens strict, ou à des raisonnements expérimentaux au sens de Claude Bernard? Quelles en sont alors les caractéristiques, voire les inflexions nécessaires pour une adaptation à leurs objets d'étude, par rapport à ce qui se fait dans les sciences de la nature? On peut rappeler brièvement ici que l'expérimentation en sciences humaines et sociales paraît soulever certains problèmes spécifiques d'ordre épistémologique, méthodologique, éthique, politique ou tout simplement pratique - quoique certains d'entre eux se posent également aux sciences biologiques et médicales. La multitude indéfinie des variables qui constituent un contexte social, la non-récurrence de leur configuration (leur historicité?) et la complexité de leurs interactions sont autant de facteurs qui paraissent de prime abord s'opposer à la simplification, à l'isolement des facteurs, à la variation contrôlée et à la réplication qui sont attendus d'un dispositif expérimental. Lorsqu'elles sont possibles, les expérimentations en sciences humaines et sociales rencontrent également souvent le problème de la « validité externe » de l'expérience, c'est-à-dire la représentativité à large échelle d'un dispositif expérimental mis en œuvre dans un contexte très singulier (et dont on peut parfois contester l'artificialité). Les expérimentations sur des sujets humains se caractérisent en outre en général, tout particulièrement en sciences humaines et sociales, par des biais résultant des interactions inconscientes et des interférences inaperçues entre les attentes ou les présupposés des expérimentateurs et ceux des sujets. Enfin, elles peuvent parfois poser des problèmes éthiques ou politiques : dommages ou risques «psychologiques » pour les sujets, problèmes 
résultant de la tromperie ou de l'injustice dans la situation expérimentale, ou encore, plus fondamentalement, difficultés que pourrait entraîner la décision de «tester» empiriquement un dispositif qui pourrait par ailleurs légitimement relever d'un choix purement politique ou déontologique [pour une brève synthèse sur ces différents problèmes, voir Dupouy 2011]. Les questions soulevées par le fait de mener une expérimentation ou un raisonnement expérimental en sciences humaines et sociales sont ainsi nombreuses et méritent d'être approfondies à la lumière de comparaisons entre disciplines.

Chacune des contributions qui suivent apporte à ce questionnement un éclairage emprunté à une discipline des sciences humaines, ou à une comparaison entre disciplines, ou encore à une technique expérimentale commune aux sciences humaines et naturelles.

L'article de Dominique Raynaud propose une étude comparative entre la sociologie et les sciences physiques. La sociologie est fréquemment perçue comme une science non expérimentale et interprétative, contrairement aux sciences physiques, qui elles seraient expérimentales et explicatives. Cet article nuance ces affirmations puisqu'il montre que le raisonnement expérimental, tel que défini par Claude Bernard et repris par Emile Durkheim, est bien présent en sociologie et en physique. L'argumentation de Dominique Raynaud se développe à travers deux études de cas : 1) la prédiction d'une déviation des rayons lumineux dans un champ de gravitation effectuée en 1919 (physique) et 2) le test de la prédiction d'un taux de cosmopolitisme de $8 \%$ de l'université de Cambridge dans la période 1250-1350 (sociologie).

C'est toujours le raisonnement expérimental au sens bernardien qui est questionné par Vincent Israël-Jost, mais cette fois-ci à travers l'étude des images produites dans le domaine des sciences biomédicales - à la frontière des sciences biologiques et des sciences humaines - à la fois pour des visées diagnostiques et expérimentales. Il s'intéresse plus précisément aux images produites grâce à la scintigraphie. Les images créées dans ce cadre à l'aide d'un instrument complexe revêtent toujours un caractère expérimental, et ce même quand elles sont utilisées à des fins diagnostiques. Vincent Israël-Jost nous montre que «l'observation est encadrée par l'expérimentation ». De ce fait, la position de Claude Bernard, qui considère le raisonnement expérimental comme une catégorie englobante (ne cherchant pas à établir une distinction nette entre observation et expérimentation), est toujours très éclairante pour comprendre les images produites dans le domaine des sciences biomédicales, et plus généralement les processus d'observation dans ce domaine.

Les deux articles suivants sont consacrés à l'économie et à ses rapports à l'expérimentation. Le premier, celui de Michel Le Du, porte sur l'économie comportementale. Ce domaine de recherche, qui se présente comme un nouveau paradigme recourant à l'expérimentation (de terrain ou en laboratoire), prétend s'affranchir des processus interprétatifs menant à la compréhension qui sont souvent considérés comme une caractéristique méthodologique propre aux sciences humaines et sociales. S'appuyant sur les travaux de Dan Ariely 
en économie comportementale (notamment), Michel Le Du formule la thèse suivante : si changement de paradigme il y a, ce n'est pas tant en raison du recours à des expérimentations qu'à cause de l'abandon de la présomption de rationalité (même s'il s'agit, selon lui, d'un abandon partiel). D'autre part, l'auteur nous montre également qu'expérimentation et compréhension ne peuvent être dissociées dans le cas de l'économie comportementale. Les décisions et les choix que l'expérimentation en économie comportementale permet d'étudier (notamment dans nos rapports à l'argent) s'expliquent par des biais qui doivent être compris. La compréhension a donc encore de beaux jours devant elle, même en économie comportementale.

Le second article sur l'économie est celui d'Arthur Jatteau. Il effectue une comparaison entre la médecine et l'économie du développement sur la question de l'utilisation des essais randomisés contrôlés (ERC). En effet, l'économie du développement s'est appropriée depuis les années 2000 ce type de méthodologie expérimentale, en s'inspirant du modèle de l'Evidence Based Medicine (EBM). Dans le domaine médical, même si les ERC sont placés très haut dans la hiérarchie des preuves, on trouve de multiples critiques très bien documentées : le fait qu'il existe d'autres méthodes, comme les méthodes observationnelles, qui sont fructueuses, le fait que nombre de traitements sont reconnus en médecine alors qu'ils ne sont pas passés sous les fourches caudines des ERC, le problème de la généralisation des résultats produits par les ERC, etc. Selon Arthur Jatteau, ces critiques, très mal connues des économistes, mériteraient d'être prises en compte en économie du développement afin de nuancer l'intérêt de la méthode en économie.

L'article de Pascal Ludwig \& Michel Matthias porte sur le rôle de l'introspection en psychologie, celle-ci étant définie comme la capacité des sujets à former des croyances sur leurs propres états mentaux. La question que posent les auteurs consiste à savoir si l'utilisation de l'introspection dans les expériences de psychologie implique pour la psychologie expérimentale de se fonder sur une méthode radicalement hétérogène à celles des sciences de la nature, au sens où l'introspection fournirait des données en première personne qui ne seraient pas publiquement observables. L'argument des auteurs pour contester à l'introspection (et par voie de conséquence à la psychologie qui l'utilise) ce statut d'exception consiste à affirmer que l'introspection en psychologie ne constitue pas une méthode de justification des hypothèses scientifiques (hypothèses qui porteraient sur des objets mentaux accessibles au seul sujet), mais une méthode de production de données (de nature verbale et comportementale) permettant au psychologue de faire des inférences sur ces objets mentaux internes. Ainsi, l'introspection jouerait plutôt le rôle d'un instrument permettant à la fois de mesurer et de manipuler les états et processus mentaux des sujets, instrument susceptible d'être calibré, à la façon dont les instruments de mesure utilisés dans les sciences de la nature peuvent l'être. De ce fait, en tant qu'instrument de mesure, l'introspection peut donner lieu à des investigations expérimentales et certaines équipes de recherche ont commencé à étudier expérimentalement l'introspection comme un mécanisme 
susceptible d'être manipulé. Si l'introspection est bel et bien un instrument de mesure, cela rapprocherait considérablement la psychologie et les sciences humaines et sociales des sciences de la nature.

L'article de Thomas Bonnin \& Jonathan Lombard propose l'étude d'une pratique scientifique en biologie de l'évolution : la phylogénie moléculaire. La phylogénie moléculaire permet de produire des arbres phylogénétiques qui nous donnent des informations sur les relations évolutives entre différentes espèces ou organismes. Les auteurs se demandent dans quelle mesure cette pratique relève, d'une part, de l'expérimentation et du style de laboratoire tels qu'ils ont été définis par Ian Hacking, et, d'autre part, d'une méthodologie typique des sciences historiques, telle que définie par Carol E. Cleland. Ils concluent que la phylogénie moléculaire ne relève à proprement parler d'aucune de ces deux méthodes, mais qu'elles sont toutes deux utiles pour définir différents aspects de cette pratique scientifique. L'analyse phylogénétique partage avec une expérimentation typique le soin apporté dans la définition du système cible et dans l'amélioration de la reproductibilité des résultats. L'idée de style de laboratoire permet de mettre en avant certaines composantes de cette pratique, et d'ouvrir la réflexion sur ses dynamiques d'autolégitimation. Par ailleurs, la phylogénie moléculaire partage avec une science historique typique l'effort pour relier des traces contemporaines par des causes communes situées dans le passé. Néanmoins, aucune de ces deux catégories n'est pleinement adéquate pour penser la démarche de l'analyse phylogénétique. Ceci amène les auteurs à rejeter l'idée d'une distinction méthodologique forte entre sciences historiques et sciences expérimentales.

Enfin, le dernier article, celui d'Yves Serra, porte sur un domaine qui, de prime abord, passe pour non scientifique et non expérimental : la philosophie morale. Mais depuis une vingtaine d'années, on constate le développement d'une philosophie morale expérimentale qui s'inspire des méthodes que l'on trouve en psychologie afin d'éclairer les débats plus classiques de la philosophie morale. La question qui se pose alors est de savoir en quoi la philosophie morale expérimentale est expérimentale. Après avoir présenté un aperçu de ce qu'est la philosophie morale expérimentale, l'auteur aborde les difficultés liées aux processus expérimentaux dans ce domaine, et à la dimension normative de la philosophie morale. Il effectue également de manière assez systématique une comparaison entre les rôles de l'expérimentation en sciences de la nature et en philosophie morale expérimentale (l'expérimentation en appui du développement des théories, le développement autonome du domaine expérimental, la spécificité de l'apport de l'expérimentation).

\section{Bibliographie}

Allamel-Raffin, Catherine \& Gangloff, Jean-Luc [2015], Some remarks about the definitions of contingentism and inevitabilism, dans : Science as 
it Could Have Been, édité par L. Soler, E. Trizio \& A. Pickering, Pittsburgh : Pittsburgh University Press, 99-113.

BAIrd, Davis [2004], Thing Knowledge. A Philosophy of Scientific Instruments, Berkeley : University of California Press.

BERNARD, Claude [1865], Introduction à l'étude de la médecine expérimentale, Paris : J. B. Baillière.

Berthelot, Jean-Michel [2001], Épistémologie des sciences sociales, Paris : PUF.

Binet, Alfred [1900], La Suggestibilité, Paris : Schleicher.

Bourgeois-Gironde, Sacha (éd.) [2012], « Et si... ? ». La cause du contrefactuel, Labyrinthe, t. 39(2), Hermann, https://journals.openedition.org/ labyrinthe/4257.

Carnap, Rudolf [1934], La tâche de la logique de la science, dans : Philosophie des sciences, édité par S. Laugier \& P. Wagner, Paris : Vrin, t. 1, 194-229, trad. S. Colas, D. Chapuis-Schmitz et P. Wagner, 2004.

Cartwright, Nancy \& Hardy, Jeremy [2013], Evidence-Based Policy : A Practical Guide to Doing it Better, Oxford : Oxford University Press.

Collins, Harry M. [1985], Changing Order : Replication and Induction in Scientific Practice, Beverly Hills : Sage Publications.

- [2001], Tacit Knowledge, Trust and the Q of Sapphire, Social Studies of Science, 31(1), 71-85, doi : 10.1177/030631201031001004.

Duflo, Esther [2009], Expérience, science et lutte contre la pauvreté, Paris : Fayard.

Dupouy, Stéphanie [2011], L'expérimentation, dans : Philosophie des sciences humaines, édité par Fl. Hulak \& Ch. Girard, Paris : Vrin, 213-241.

Durkheim, Émile [1894], Les Règles de la méthode sociologique, Paris : PUF, 1967.

Franklin, Alan [1990], Experiment, Right or Wrong?, Cambridge : Cambridge University Press.

Franklin, Allan [1986], The Neglect of Experiment, Cambridge : Cambridge University Press.

- [2016], What makes a "good" experiment?, The British Journal for the Philosophy of Science, 32(4), 367-374, doi : 10.1093/bjps/32.4.367. 
Galavotti, Maria Carla (éd.) [2004], Observation and Experiment in the Natural and Social Sciences, New York : Kluwer Academic Publishers.

Galison, Peter [1987], How Experiments End, Chicago : University of Chicago Press, trad. fr. par B. Nicquevert, Ainsi s'achèvent les expériences, Paris : La Découverte, 2002.

[1997], Image and Logic, Chicago : University of Chicago Press.

Gooding, David, PInch, Trevor et al. [1989], The Uses of Experiment : Studies in the Natural Sciences, Cambridge : Cambridge University Press.

Gueron, Judith M. \& Rolston, Howard [2013], Fighting for Reliable Evidence, New York : Russell Sage Foundation Publications.

HACKInG, Ian [1983], Representing and Intervening : Introductory Topics in the Philosophy of Natural Science, Cambridge : Cambridge University Press, trad. fr. par B. Ducrest, Concevoir et experimenter, Paris : Christian Bourgois, 1989.

— [1992], The self-vindication of the laboratory sciences, dans : Science as Practice and Culture, édité par A. Pickering, Chicago : University of Chicago Press, 29-64.

[1999], The Social Construction of What?, Cambridge : Cambridge University Press.

- [2005], Les philosophes de l'expérience, Tracés, 9, 67-82, doi : 10.4000/ traces.201, trad. fr de Philosophers of Experiment, PSA : Proceedings of the Biennial Meeting of the Philosophy of Science Association, 1988/2, 147-156, 1988.

HARRÉ, Rom [2003], The materiality of instruments in a metaphysics of experiment, dans : The Philosophy of Scientific Experimentation, édité par H. Radder, Pittsburgh : University of Pittsburgh Press, 19-38.

Hollis, Martin [2002], The Philosophy of Social Science. An introduction, Cambridge : Cambridge University Press.

Hon, Giora [1989], Towards a typology of experimental errors : An epistemological view, Studies in History and Philosophy of Science Part A, 20(4), 469-504, doi : 10.1016/0039-3681(89)90020-4.

— [2003], The idols of experiment. Transcending the "etc. list", dans : The Philosophy of Scientific Experimentation, édité par H. Radder, Pittsburgh : University of Pittsburgh Press, 174-197.

Knobe, Joshua Michael \& Nichols, Shaun (éds.) [2008], Experimental Philosophy, Oxford : Oxford University Press. 
Kunn, Thomas [1962], The Structure of Scientific Revolutions, Chicago : University of Chicago Press.

Labrousse, Agnès [2010], Nouvelle économie du développement et essais cliniques randomisés : une mise en perspective d'un outil de preuve et de gouvernement, Revue de la régulation, 7, 2-32, doi : 10.4000/regulation.7818.

Latour, Bruno \& Woolgar, Steve [1979], Laboratory Life. The Construction of Laboratory Facts, Sage, trad. fr par M. Biezunski, La Vie de laboratoire, Paris : La Découverte, 1996.

Martin, Michael [1994], The philosophical importance of the Rosenthal Effect, dans : Readings in the Philosophy of Social Science, édité par M. Martin \& L. C. Mc Intyre, Cambridge : MIT Press, 585-596.

Mayo, Elton [1933], The Human Problems of an Industrial Civilization, New York : Routledge.

Mesure, Sylvie \& Savidan, Patrick [2006], Dictionnaire des sciences humaines, Paris : PUF.

Monnier, Éric [1992], Évaluations de l'action des pouvoirs publics, Paris : Economica.

Monteix, Nicolas \& Poidevin, Aurélien (éds.) [2019], L'Expérimentation, un matériau de l'histoire, Rouen; Le Havre : Presses Universitaires de Rouen et du Havre.

NADEAu, Robert [1999], Vocabulaire technique et analytique de l'épistémologie, Paris : Presses Universitaires de France.

NAGEL, Ernst [1961], The Structure of Science, New York : Routledge.

Ogien, Ruwen [2001], Philosophie des sciences sociales, dans : Epistémologie des sciences sociales, édité par J.-M. Berthelot, Paris : Presses Universitaires de France, 521-575.

—_ [2011], L'Influence de l'odeur des croissants chauds sur la bonté humaine et autres questions de philosophie morale expérimentale, Paris : Grasset.

ORNe, Martin T. [1962], On the social psychology of the psychological experiment : With particular reference to demand characteristics and their implications., American Psychologist, 17(11), 776-783, doi : 10.1037/ h0043424.

Passeron, Jean-Claude [1991], Le Raisonnement sociologique. L'espace nonpoppérien du raisonnement naturel, Paris : Nathan.

Pickering, Andrew [1984], Constructing Quarks, Chicago : University of Chicago Press. 
Pickering, Andrew (éd.) [1992], Science as Practice and Culture, Chicago : University of Chicago Press.

Pickering, Andrew [1995], The Mangle of Practice. Time, Agency, and Science, Chicago : University of Chicago Press.

Popper, K. [1934], The Logic of Scientific Discovery, Londres; New York : Routledge Classics.

[1965], Conjectures et réfutations, Paris : Payot, trad. fr. par M. I. et M. B. de Launay, 1985.

RADDER, Hans [2003], The Philosophy of Scientific Experimentation, Pittsburgh : University of Pittsburgh Press.

Rosenthal, Robert A., Jacobson, Leonore et al. [1971], Pygmalion à l'école, Paris : Casterman, trad. fr. par S. Audebert et Y. Rickards, titre original Pygmalion in the Classroom, New York : Holt Rinehart and Winston,1968.

SERrA, Daniel [2015], Histoire de l'économie expérimentale, Saarbruck : Éditions Universitaires Européennes.

SOLER, Léna [2009], Introduction à l'épistémologie, Paris : Ellipses.

Soler, Léna, Zwart, Sjoerd et al. [2014], Science After the Practice Turn in the Philosophy, History, and Social, Studies of Science, Londres : Routledge.

Turner, Stephen P. \& Risjord, Mark W. [2007], Philosophy of Anthropology and Sociology, Amsterdam : Elsevier. 